\title{
ULTRA-RELATIVISTIC RADIAL EXPANSION OF PERFECT FLUID WITH SHOCK WAVES
}

\author{
V. I. Zhdanov, M. S. Borshch \\ National Taras Shevchenko University of Kyiv \\ (Received February 16, 2006; in final form - October 31, 2006)
}

\begin{abstract}
We obtain approximate general solutions of hydrodynamical equations, which describe spherical or cylindrical expansion of perfect fluid with shock waves in ultra-relativistic limit. The equation of state is $p=k(\varepsilon-\rho), \varepsilon$ is the energy density and $\rho$ is the baryon mass density. The solutions involve functional degrees of freedom inherent in partial differential equations. Internal shock wave between two domains of ultra-relativistic flow and external shock propagating into a static medium are considered.
\end{abstract}

Key words: relativistic hydrodynamics, shock waves, gamma-ray bursts

PACS number(s): 47.75.+f, 98.70.Rz

\section{INTRODUCTION}

Relativistic hydrodynamics is widely used in the analysis of gamma-ray bursts and other powerful explosions in the Universe. A typical problem concerning these phenomena deals with the investigation of ultra-relativistic radial expansion of the perfect fluid [1]. There are special analytical solutions of this problem in case of spherical or cylindrical explosions [2,3]. A complete qualitative investigation of self-similar solutions to relativistic hydrodynamical equations with the equation of state $p=k \varepsilon$ can be found in [4]. The Blandford \& McKee solution [2] is most extensively used in astrophysical applications. This solution describes an ultra-relativistic outflow of perfect fluid following a shock wave.

All the above solutions deal with special (self-similar) fluid motions that may be described by ordinary differential equations; these solutions may be restricted by a finite number of parameters. However, observations reveal more complicated picture of hydrodynamical outflow that may include, e.g., interacting shock waves [5]. Therefore, it is of interest to study solutions having sufficient functional freedom, which is appropriate to partial differential equations. In our earlier paper [6] we found solutions that describe ultra-relativistic radial expansion of perfect fluid in case of a continuous flow (without shocks). These solutions contain arbitrary functions to present a sufficiently general form of ultra-relativistic limit. In the present we paper use these results to obtain solutions describing ultra-relativistic expansion in the presence of shocks that separate regions of a continuous flow.

According to [2] we use the model equation state (EOS)

$$
p=k(\varepsilon-\rho),
$$

where $p$ is the pressure, $k=$ const, $\varepsilon$ and $\rho<\varepsilon$ are invariant thermodynamical parameters defined in the rest frame of a fluid element: $\varepsilon$ is the local energy density, $\rho$ is the baryon proper mass density; the light speed $c=1$.
This equation of state is used, e.g., in considerations of ultra-relativistic shock propagation in cold matter [2], when ahead of the shock there is a static medium with zero pressure $p=0$. In Section II we obtain solutions describing the ultra-relativistic continuous flow in an implicit form. They are reduced to explicit solutions in case of a hot matter approximation, when $\rho \ll \varepsilon$ and $p \approx k \varepsilon$. These results are applied to an "internal" shock wave between two domains of ultra-relativistic flow (Section III) and external shock propagating in a static medium (Section IV).

\section{ULTRA-RELATIVISTIC APPROXIMATION}

The relativistic equations of a perfect fluid follow from the conservation equations $T^{\mu \nu}{ }_{, \nu}=0$ involving the energy-momentum tensor of perfect fluid $T^{\mu \nu}=$ $(p+\varepsilon) u^{\mu} u^{\nu}-p g^{\mu \nu}$. The other set of equations describes conservation of baryonic charge $\left(\rho u^{\mu}\right),{ }_{\mu}=0$. Further we use the same variables as in [6]:

$$
u^{t}=u+1 /(4 u), \quad u^{r}=u-1 /(4 u),
$$

where $u^{t}$ and $u^{r}$ are the four-velocity components, and $\alpha=t-r, \beta=t+r, r$ is the radial variable in case of a cylindrical $(J=1)$ and spherical $(J=2)$ expansion. In ultra-relativistic limit $u^{r} \approx u^{t} \approx u \gg 1$. The hydrodynamical equations in these variables may be written as follows [6]:

$$
\begin{gathered}
\frac{\partial}{\partial \beta}(\varepsilon-p)+\frac{\partial}{\partial \alpha}\left(\frac{\varepsilon+p}{4 u^{2}}\right)=-\frac{J(\varepsilon+p)}{\beta-\alpha}\left(1-\frac{1}{4 u^{2}}\right) \\
\frac{\partial}{\partial \beta}\left[u^{2}(\varepsilon+p)\right]+\frac{\partial}{\partial \alpha}\left(\frac{\varepsilon-p}{4}\right)=-\frac{J(\varepsilon+p)}{\beta-\alpha}\left(u^{2}-\frac{1}{4}\right) .
\end{gathered}
$$

The baryonic charge conservation equations 


$$
\frac{\partial}{\partial t}\left(\rho u^{t} r^{J}\right)+\frac{\partial}{\partial r}\left(u^{r} r^{J} \rho\right)=0
$$

after analogous substitution of variables (2) take on the form

$$
\frac{\partial}{\partial \beta}\left[\rho u(\beta-\alpha)^{J}\right]+\frac{1}{4} \frac{\partial}{\partial \alpha}\left[\frac{\rho(\beta-\alpha)^{J}}{u}\right]=0 .
$$

The system (3)-(5) is closed by the EOS (1). In the presence of shocks equations (3)-(5) must be supplemented with relations on discontinuities of the fluid flow (see Section III)

Further we deal only with the main orders of ultrarelativistic approximation: we retain the terms containing the higher powers of $u \gg 1$, in particular we neglect the terms $\sim \frac{\partial}{\partial \alpha}$ in Eq. (4) and Eq. (5). The resulting equations can be easily solved

$$
\begin{gathered}
u^{2}(p+\varepsilon)(\beta-\alpha)^{J}=g(\alpha), \\
u \rho(\beta-\alpha)^{J}=h(\alpha),
\end{gathered}
$$

where $g(\alpha)$ and $h(\alpha)$ are arbitrary functions.

This enables us to express $\varepsilon$ and $\rho$ in terms of $u$. Substitution into Eq. (3) after analogous approximation leads to an equation for $u$

$$
\frac{(1-k) g(\alpha)+k u h(\alpha)}{u[g(\alpha)-u h(\alpha)]} \frac{\partial u}{\partial \beta}=\frac{J k}{\beta-\alpha}
$$

whence

$$
\frac{u^{1-k}}{g(\alpha)-u h(\alpha)}=\psi(\alpha)(\beta-\alpha)^{J k},
$$

$\psi(\alpha)$ being an arbitrary function. The left-hand side is a monotonous function of $u$ and there may be only one solution of Eq. (8) with respect to $u$. Thus, equations $(6),(7),(8)$ present a solution of hydrodynamical equations with the equation of state (1) in an implicit form.

In this section we confine ourselves to the case of hot matter with $\varepsilon \gg \rho$. In this case $g(\alpha) \gg u h(\alpha)$ and the solution may be written explicitly

$$
\begin{gathered}
\varepsilon=\frac{F(\alpha)}{r^{a}}, \quad a=\frac{J(1+k)}{1-k} \\
u^{2}=G(\alpha) r^{b}, \quad b=\frac{2 J k}{1-k} \\
\rho=H(\alpha) r^{-\frac{J}{1-k}},
\end{gathered}
$$

where $r=(\beta-\alpha) / 2$,

$$
\begin{gathered}
F=\left[(1+k)\left(2^{J} g\right)^{\frac{1+k}{1-k}} \psi^{\frac{2}{1-k}}\right]^{-1}, \quad G=\left[2^{J k} g \psi\right]^{\frac{2}{1-k}}>0, \\
H=h\left(2^{J} g \psi\right)^{-\frac{1}{1-k}} .
\end{gathered}
$$

At this point we can leave behind the functions $g, h, \psi$ and deal with the solution (9)-(11) in terms of independent arbitrary positive functions $G(\alpha), F(\alpha), H(\alpha)$. This agrees with the first order approximation of solution [6] of hydrodynamical equations obtained in the case of EOS $p=k \varepsilon$. However, from Eqs. (9) and (11) we observe that $\varepsilon$ decreases faster than $\rho$, so in the course of time these values become comparable.

Using (9)-(11), the conditions $u \gg 1$ and $\varepsilon \gg \rho$ can be written as

$$
\begin{gathered}
r^{b} G(\alpha) \gg 1, \\
r^{\frac{J k}{1-k}} H(\alpha) \ll F(\alpha) .
\end{gathered}
$$

Condition (13) may be valid only for limited values of $r$ and $t$; this is natural because the expanding fluid gets colder and the approximation of hot matter fails with time.

Additional conditions that validate the ultrarelativistic approximations may be obtained by comparing the retained and omitted terms in Eqs. (3)-(5). This gives

$$
\left|\frac{\partial}{\partial \alpha}\left(\frac{\varepsilon}{u^{2}}\right)\right| \ll \frac{\varepsilon}{\beta-\alpha}, \quad\left|\frac{\partial \varepsilon}{\partial \alpha}\right| \ll \frac{\varepsilon u^{2}}{\beta-\alpha},
$$

$$
\left|\frac{\partial(\rho / u)}{\partial \alpha}\right| \ll \frac{\rho u}{\beta-\alpha}
$$

Using Eqs. (9)-(11) these inequalities may be represented as follows

$$
r^{b-1} G(\alpha) \gg \max \left\{\left|\frac{F^{\prime}(\alpha)}{F(\alpha)}\right|,\left|\frac{G^{\prime}(\alpha)}{G(\alpha)}\right|\right\},
$$

and

$$
r^{b-1} G(\alpha) \gg\left|\frac{H^{\prime}(\alpha)}{H(\alpha)}\right|
$$

Inequalities (14) and (15) justify approximations in Eqs. (3), (4) and Eq. (5), respectively. They put limits on the domain of validity of the approximations. These inequalities may be fulfilled, e.g., in case of a powerlaw dependence of $F(\alpha), G(\alpha)$ and $H(\alpha)$ within an expanding shell behind the shock front: in this case for $G(\alpha)=C_{0} \alpha^{-n}, n>1$ inequalities (14), (15) reduce to

$$
C_{0} r^{b-1} \gg \alpha^{n-1} .
$$

Note that if $k>(2 J+1)^{-1}(b>1)$, then inequality (16) may hold for $r \rightarrow \infty$ for a bounded $\alpha$. In this case the solution describes a spherical shell moving outwards almost with the light speed and its energy remains finite for $t \rightarrow \infty$ until (13) is violated. 


\section{INTERNAL SHOCK WAVE}

Using known relations on relativistic shocks [7] we have for the shock trajectory $r=R(t)$

$$
\dot{R}=\frac{v_{s}+v_{0}}{1+v_{s} v_{0}}, \quad v_{s}^{2}=\frac{\left(p-p_{0}\right)\left(p_{0}+\varepsilon\right)}{\left(\varepsilon-\varepsilon_{0}\right)\left(p+\varepsilon_{0}\right)},
$$

here $v_{0}$ is the fluid velocity ahead of the shock (in a "laboratory frame"), $v_{s}$ is the shock velocity with respect to the fluid ahead of the shock; $p, \varepsilon$ correspond to the state behind the shock and $p_{0}, \varepsilon_{0}$ correspond to the state ahead of the shock.

The equation for the fluid velocity behind the shock is [7]

$$
v=\frac{v_{\mathrm{fl}}+v_{0}}{1+v_{\mathrm{fl}} v_{0}}, \quad v_{\mathrm{fl}}^{2}=\frac{\left(\varepsilon-\varepsilon_{0}\right)\left(p-p_{0}\right)}{\left(p+\varepsilon_{0}\right)\left(p_{0}+\varepsilon\right)},
$$

$v_{\mathrm{fl}}$ being the fluid velocity behind the shock with respect to the fluid ahead of the shock.

The relativistic Hugoniot-Taub adiabat [7] relates the thermodynamical quantities on both sides of the shock. We use the equation of the adiabat in the form [8]:

$$
\frac{\rho^{2}}{\rho_{0}^{2}}=\frac{(p+\varepsilon)\left(p_{0}+\varepsilon\right)}{\left(p_{0}+\varepsilon_{0}\right)\left(p+\varepsilon_{0}\right)} .
$$

In case of the EOS (1) if, e.g., in the initial state $\rho_{0}=\varepsilon_{0}\left(p_{0}=0\right)$, then for $\varepsilon>\varepsilon_{0}$ we have the only positive solution of Eq. (19), such that $\rho \neq \varepsilon$ :

$$
\rho=(2 k)^{-1}\left[\varepsilon_{0}+\sqrt{\varepsilon_{0}^{2}+4 k(1+k) \varepsilon_{0} \varepsilon}\right] .
$$

Further we again confine ourselves to the case $\rho \ll \varepsilon$, $p \approx k \varepsilon$ in the ultra-relativistic flow. This follows automatically from $(20)$, if the shock is strong $\left(\varepsilon \gg \varepsilon_{0}\right)$ :

$$
\rho \approx \sqrt{\frac{1+k}{k} \varepsilon_{0} \varepsilon} \ll \varepsilon .
$$

In a more general case using $(p+\varepsilon) /\left(p+\varepsilon_{0}\right)<\varepsilon / \varepsilon_{0}$ for $\varepsilon>\varepsilon_{0}$ and $p \geq 0$, we have from Eq. (19)

$$
\rho<\rho_{0} \frac{\varepsilon}{\varepsilon_{0}} .
$$

Therefore if $\rho_{0} \ll \varepsilon_{0}$ ahead of the shock, then an analogous relation necessarily is valid behind the shock: $\rho \ll \varepsilon$.

Consider the case, when the shock separates two ultrarelativistic flows (Fig. 1); the flow behind the shock is described by (9)-(11) and the flow ahead of the shock is described by the relations

$$
u^{2}=G_{0}(\alpha) r^{b}, \quad \varepsilon=F_{0}(\alpha) r^{-a}, \quad \rho=H_{0}(\alpha) r^{-\frac{J}{1-k}} .
$$

Approximation (22) will be valid in the domain of variables $\alpha, r$, where the functions $F_{0}, G_{0}, H_{0}$ satisfy the analogous inequalities as (12), (13) and (14), (15) for the functions $F, G, H$.
Taking into account that $v_{0} \approx 1$, we have from $(17)$ an equation for the shock trajectory $\alpha_{s}(t)=t-R(t)$

$$
\dot{\alpha}_{s}=\frac{1-k^{2}}{2 R^{b} G_{0}\left(\alpha_{s}\right)}[\sqrt{k \mu+1}+\sqrt{k(\mu+k)}]^{-2},
$$

where $\mu=F\left(\alpha_{s}\right) / F_{0}\left(\alpha_{s}\right)$.

Two other relations must be satisfied for $\alpha=\alpha_{s}(t)$ : Eq. (18) for the fluid velocity yields

$$
\frac{G}{G_{0}}=\frac{[\sqrt{(k \mu+1)(\mu+k)}+\sqrt{k}(\mu-1)]^{2}}{(k+1)^{2} \mu},
$$

and (19) for the shock adiabat yields

$$
\frac{H}{H_{0}}=\left(\mu \frac{\mu+k}{k \mu+1}\right)^{1 / 2} .
$$

These relations are especially simple, if $\mu \gg 1$, that is the shock is ultra-relativistic also in the frame of the medium " 0 " ahead of the shock:

$$
\begin{gathered}
\dot{\alpha}_{s}=\frac{1-k^{2}}{8 k G_{0}\left(\alpha_{s}\right) \mu\left(\alpha_{s}\right) R^{b}}, \\
\frac{G\left(\alpha_{s}\right)}{G_{0}\left(\alpha_{s}\right)}=\frac{4 k \mu\left(\alpha_{s}\right)}{(1+k)^{2}}, \\
\frac{H\left(\alpha_{s}\right)}{H_{0}\left(\alpha_{s}\right)}=\left[\frac{\mu\left(\alpha_{s}\right)}{k}\right]^{1 / 2}
\end{gathered}
$$

One may require Eqs. (24), (25) be valid for all $\alpha$. However, in fact (24) and (25) restrict $G(\alpha), H(\alpha)$ for the given functions $F, F_{0}, G_{0}, H_{0}, \rho_{0}$ only in the domain, where $\alpha_{s}(t)$ varies for $t>t_{0}$. For strong shocks this may be very small interval on $\alpha$-axis. Indeed, if, e.g. $G_{0}\left(\alpha_{s}\right) \mu\left(\alpha_{s}\right) \sim$ $\alpha^{n}, n>0$, then Eq. (26) yields $\alpha^{n+1} \sim$ const $+t^{1-b}$ that is for $b>1$ and sufficiently large $t$ the shock moves almost with the light speed, and the value of $\alpha_{s}$ is "frozen".

\section{EXTERNAL SHOCK WAVE}

Let the fluid ahead of the shock be at rest (see Fig. 2). The energy profile before the shock is $\varepsilon_{0}(R)$ and the pressure is $p_{0}=0, \varepsilon_{0}=\rho_{0}$. Let $\varepsilon \gg \varepsilon_{0}$ behind the shock. Then (see (21)) $\rho \ll \varepsilon$, so from Eq. (17) we have

$$
\dot{\alpha_{s}}=\frac{(1-k) \varepsilon_{0}}{2 k \varepsilon}=\frac{(1-k) \varepsilon_{0}(R)}{2 k F\left(\alpha_{s}\right)} R^{a}
$$

The other condition (18) for $\varepsilon \gg \varepsilon_{0}$ yields

$$
u^{2}=\frac{k \varepsilon}{(1+k) \varepsilon_{0}}
$$

whence

$$
G\left(\alpha_{s}\right)=\frac{k F\left(\alpha_{s}\right)}{(1+k) R^{a+b} \varepsilon_{0}(R)} .
$$


We shall see that due to Eqs. (29) and (30) there is a drastic variation of $\alpha_{s}$ in a small region leading to violation of the condition (14). For $\varepsilon \gg \varepsilon_{0}$ Eq. (29) yields $d R \approx d t$. Then from (30) and (29) it follows that

$$
\begin{aligned}
& \frac{G^{\prime}\left(\alpha_{s}\right)}{G\left(\alpha_{s}\right.}=\frac{F^{\prime}\left(\alpha_{s}\right)}{F\left(\alpha_{s}\right)}-\left[\frac{a+b}{R}+\frac{\varepsilon_{0}^{\prime}(R)}{\varepsilon_{0}(R)}\right] \frac{d R}{d \alpha_{s}} \approx \\
\approx & \frac{F^{\prime}\left(\alpha_{s}\right)}{F\left(\alpha_{s}\right)}-\left[\frac{a+b}{R}+\frac{\varepsilon_{0}^{\prime}(R)}{\varepsilon_{0}(R)}\right] \frac{2 k F\left(\alpha_{s}\right)}{(1-k) \varepsilon_{0}(R) R^{a}} .
\end{aligned}
$$

Typically (e.g., for $\frac{d}{d R}\left[\ln \left(\varepsilon_{0}(R) R^{a+b}\right)\right] \sim R^{-1}$ ) the second item in the latter expression is of the same order as

$$
u^{2} R^{-1}=G\left(\alpha_{s}\right) R^{b-1}=\frac{k F\left(\alpha_{s}\right)}{(1+k) \varepsilon_{0}(R) R^{a+1}} .
$$

Thus, condition (14) is not valid except for the case when

$$
\varepsilon_{0}(R) R^{a+b}=E_{0}=\text { const. }
$$

In fact conditions (14) may be weakened. This, however, requires a more detailed additional consideration, which is not presented here. We only note that approximate relations (9), (11) represent correct qualitative behavior of solutions in the context of external shock wave. But in this paper we consider only the case when the above ultra-relativistic approximation works. Using (31) we have for $\alpha_{s} \ll t \rightarrow \infty$

$$
\dot{\alpha_{s}}=\frac{(1-k) E_{0}}{2 k F\left(\alpha_{s}\right) R^{b}} \approx \frac{(1-k) E_{0}}{2 k F\left(\alpha_{s}\right) t^{b}},
$$

so the shock trajectory is given implicitly as

$$
\int_{0}^{\alpha_{s}} F(\alpha) d \alpha=-\frac{(1-k) E_{0}}{2 k(b-1) t^{b-1}} .
$$

There is an additional relation imposed by shock adiabat (19), which yields

$$
\frac{\rho}{\rho_{0}}=\left[\frac{(k+1) \varepsilon}{k \varepsilon_{0}}\right]^{1 / 2} .
$$

This defines $H(\alpha)$ through $F(\alpha)$ and $\varepsilon_{0}(r)$ :

$$
H\left(\alpha_{s}\right)=\left[\frac{k+1}{k} \varepsilon_{0}(R) R^{J} F\left(\alpha_{s}\right)\right]^{1 / 2} .
$$

In view of (35) analogous considerations show that condition (15) is valid only if $\varepsilon_{0}(R) R^{J}=$ const; however, this is not compatible with Eq. (31) except trivial case of $k=0$. So, to avoid condition (15) we return to exact for the equation (5) that can be rewritten as

$$
\frac{\partial Y}{\partial \beta}+\frac{1}{4 u^{2}} \frac{\partial Y}{\partial \alpha}+\frac{\partial}{\partial \alpha}\left(\frac{1}{4 u^{2}}\right)=0,
$$

where $Y=\ln \left(\rho u r^{J}\right)$. Using (10) we have

$$
\frac{\partial Y}{\partial \beta}+\frac{2^{b-2}}{(\beta-\alpha)^{b} G(\alpha)} \frac{\partial Y}{\partial \alpha}+\frac{\partial}{\partial \alpha}\left(\frac{2^{b-2}}{(\beta-\alpha)^{b} G(\alpha)}\right)=0 .
$$

This equation may be solved in the domain $\alpha \ll \beta$ (behind the shock) under assumption that

$$
\left|\frac{G^{\prime}(\alpha)}{G(\alpha)}\right| \gg \frac{b}{\beta}
$$

This inequality is fulfilled in the above domain, e.g., in case of $G(\alpha) \sim \alpha^{-n}$; we remind that (38) must be compatible with (16). Under this condition (37) yields

$$
\beta^{b} \frac{\partial Y}{\partial \beta}+\frac{2^{b-2}}{G(\alpha)} \frac{\partial Y}{\partial \alpha}+\frac{\partial}{\partial \alpha}\left(\frac{2^{b-2}}{G(\alpha)}\right)=0 .
$$

Change of variables $\alpha, \beta$ to $\alpha, \xi$, where

$$
\xi=\int G(\alpha) d \alpha+\frac{2^{b-2}}{(b-1) \beta^{b-1}},
$$

leads to the equation

$$
\frac{\partial Y}{\partial \alpha}=\frac{G^{\prime}(\alpha)}{G(\alpha)}
$$

Then

$$
\rho u r^{J}=\exp (Y)=G(\alpha) F(\xi),
$$

$F$ being an arbitrary function. Under condition (16) we expect that

$$
\left.\mid \int G(\alpha) d \alpha\right) \mid \gg \frac{1}{(b-1) \beta^{b-1}} .
$$

Then $F(\xi)$ is approximately a function on $\alpha$ only and we again come to (7) and then to (11) without using (15).

\section{CONCLUSIONS}

We have obtained approximate general solutions of equations of the relativistic perfect fluid motion with the equation of state $p=k(\varepsilon-\rho)$. The solutions describe a spherical $(J=2)$ or cylindrical $(J=1)$ ultra-relativistic expansion of the fluid shells; they involve three arbitrary functions of one variable necessary to provide sufficient functional freedom. In the case of hot matter $\varepsilon \gg \rho$ the solution has rather a simple explicit form (9)-(11). The domain, where the approximations are valid, is given by inequalities (12), (13), (14), (15). The approximations may be used until $\rho \ll \varepsilon$ ceases to be valid; of course the equation of state must be modified as the fluid expands.

We constructed solutions containing "internal" shock waves that separate two domains of ultra-relativistic flow, and "external" shock propagating into static medium. In the case of "internal" shocks our result is sufficiently general. However, in the case of "external" shock our analysis is valid only if the matter density ahead of the shock has some special power-law form; e.g., for $k=1 / 3$ and $J=2$ one needs $\varepsilon_{0}(r) \sim r^{6}$. Outside this case an additional consideration is needed to prove the validity of approximations; we expect, however, that this consideration will not change essentially the qualitative behavior of solutions. 
[1] P. Meszaros, Ann. Rev. Astron. Astrophys. 40, 137 (2002).

[2] R. D. Blandford, C. F. McKee, Phys. Fluids 19, 1130 (1976).

[3] R. Sari, preprint astro-ph/0505174 (2005).

[4] N. R. Sibgatullin, Oscillations and Waves in Strong Gravitational and Electromagnetic Fields (Nauka, Moscow,
1984).

[5] T. Piran, E. Nakar, preprint astro-ph/0202403 (2002).

[6] V. I. Zhdanov, M. S. Borshch, J. Phys. Stud. 9, 233 (2005).

[7] L. D. Landau, E. M. Liftshits, Theoretical Physics. Vol. VI: Hydrodynamics (Nauka, Moscow, 1986) [in Russian].

[8] K. A. Bugaev, M. I. Gorenstein, V. I. Zhdanov, Z. Phys. C 39, 365 (1988).

\title{
УЛЬТРАРЕЛЯТИВІСТСЬКЕ РАДІАЛЬНЕ РОЗШИРЕННЯ ІДЕАЛЬНОЇ РІДИНИ З УДАРНИМИ ХВИЛЯМИ
}

\author{
В. І. Жданов, М. С. Борщ \\ Київсъкий начіональний університет імені Тараса Шевченка, \\ Астрономічна обсерваторія, вул. Обсерваторна, 3, Київ, 04053, Украӥна
}

Отримано наближені розв'язки рівнянь гідродинаміки, що описують сферичне або циліндричне розширення ідеальної рідини з ударними хвилями в ультрарелятивістській межі. Рівняння стану є $p=k(\varepsilon-\rho)$, де $\varepsilon$ - густина енергії, $\rho$ густина маси баріонів. Розв'язки містять функціональні степені вільности, притаманні для рівнянь з частинними похідними. Розглянуто внутрішні ударні хвилі між двома ділянками ультрарелятивістських течій та зовнішні ударні хвилі, що поширюються в ділянку статичного середовища. 